\title{
Intrinsic and Extrinsic Risk Factors for Lateral Ankle Sprain: A Literature Review
}

\author{
Miguel Ângelo de Castro Correia ${ }^{1 *}$ and João Torres ${ }^{2}$
}

${ }^{1}$ Faculty of Medicine, University of PORTO, Portugal

${ }^{2}$ Orthopaedics Department, Faculty of Medicine, University of PORTO, Portugal

\section{Introduction}

Ankle injuries are very common, specially sprained ankles [1]. Fong, et al. [1] reported that ankle sprain was the major ankle injury in 33 of 43 sports. In high impact, contact and pivoting activities, as well as in jumping and landing, a high proportion of the injuries will affect the lower limb, namely the ankle joint [2-4].

Ankle sprain can injure the medial ligaments, lateral ligaments, or the syndesmotic tibio-fibular joint. Respectively, these injuries are referred as medial, lateral or high ankle sprains. Lateral ankle sprains are more common, and athlete's are at a significantly higher risk of sustaining one [5]. This review will focus primarily on lateral ankle sprains, which from now on, will be referred only as ankle sprains.

Very often, an exaggerated supination and inversion of the foot, with external rotation of the tibia leads to this injury [6]. This mechanism generates tension that stretches the ligaments beyond their tensile strength, leading to damage [6]. Classically, this damage is divided in three grades and the patient usually has pain with weight bearing, ecchymosis and swelling, progressively increasing with the grade [7].

Most of the times, the treatment for a sprained ankle is conservative $[2,6,8,9]$, rarely including surgery [10]. Even though ankle sprains tend to be considered "easy to treat", sometimes a bad outcome may be present [11]. According to the severity of the tear, the patient might have different grades of short-and long-term disabilities.

As mentioned above, ankle sprain treatment is usually conservative. Nonetheless, considering the high incidence of this injury, and the negative impact that it might have on personal, professional and leisure activity, it's easy to understand why medical teams make a huge effort trying to prevent it. A thorough understanding of the risk factors behind ankle sprain is mandatory to create proper education and prevention programs, either in non-professional and professional sports activities. This literature review intends to summarize and condense the available scientific evidence up to the date.

\section{Methods}

We searched MEDLINE on 24 September 2018 using the keywords ("sprained ankle" OR "ankle sprain") and ("risk" OR "predictive" OR "prevention") and the filter "Article - Systematic Review". Studies investigating risk or predictive factors for lateral ankle sprain, as well as lateral ankle sprain prevention, were included. There was no limitation based on physical activity, sports, age or study publication date. Studies were excluded based on title and abstract reading. If any doubt remained, the article full text was read. Literature [12] suggests some risk factors for ankle sprain which are not yet covered by a systematic review, and further reading on these risk factors was made.

\section{Results}

Our search led to sixty-two articles. Nine articles were excluded because they covered ankle sprain treatment or rehabilitation. Three articles were excluded, as they covered chronic ankle instability. Four further articles were excluded because, besides covering ankle sprain, they didn't study any risk factor or preventive measure. We also excluded four more articles: One about upper ankle sprain; one that covered the fracture risk during an ankle sprain; one that reported findings on anterior cruciate ligament; and the last, which was withdrawn from the database. Finally, 42 studies were included in our review.

\section{Discussion}

Williams [13] proposed a classification for injuries' mechanism, which we adapted, dividing risk factors into intrinsic (one's characteristics) and extrinsic (external causative agent).

*Corresponding author: Miguel Ângelo de Castro Correia, MD, Faculty of Medicine- University of PORTO, Portugal

Accepted: September 12, 2019

Published online: September 14, 2019

Citation: Correia MAC, Torres J (2019) Intrinsic and Extrinsic Risk Factors for Lateral Ankle Sprain: A Literature Review. Arch Sports Med 3(2):172-177 


\section{Intrinsic risk factors}

Gender: Most of the studies didn't show any significant difference in ankle sprain incidence between male and female. Nonetheless Doherty, et al. [5] reviewed and meta-analyzed 181 prospective studies and found that women were at a higher risk of sustaining an ankle sprain, compared to men (13.6 vs. 6.94 sprains per 1000 exposures). To our knowledge, this was the first systematic review including this number of articles and finding differences between genders. However, the reason behind this difference is still unknown. Further studies should target if sex disparities are related to different intensities and behaviors during sports practice, or whether the higher risk in women is related to morphophysiological female characteristics.

Age: Once again, Doherty $C$, et al. [5] is paving the way to clear if age is related to ankle sprains. Their results showed a higher risk of ankle sprains in children compared to adolescents (2.84 vs. 1.94 per 1000 exposures), and adolescents compared with adults (1.94 vs. 0.72 per 1000 exposures). Other author's researches [14-16] showed similar results to Doherty, however not all studies are unanimous [17].

The higher incidence of sprain ankles in younger athletes could be explained by a not fully developed sensorimotor function [18]. Furthermore, the need for injury prevention is mandatory, because injury at a young age can have a negative impact on children's activity and may lead to long term complications [18-20].

Height, weight and body mass index: Height and weight have been implicated as risk factors for sprained ankles [21], because an increase in either of them, proportionally increases the magnitude of inversion torque that must be resisted by the ankle-complex, when the foot is in an at-risk position [12]. However, many studies didn't find an independent significant association between ankle sprain and height or weight $[4,17,22-24]$.

Even though weight and height individually haven't been clearly defined as risk factors for ankle sprain, some studies showed significant correlation between BMI and sprained ankle $[15,25]$. In a recent systematic review with metaanalysis [26], greater BMI was found in the injury group.

The fact that higher BMI is significantly related to ankle sprain, while height and weight aren't, suggests that BMI is a more reliable measure to express one's body constitution. Weight and height should not be seen separately, because they are inextricable.

Muscle strength and muscle-reaction time: The ankle joint is stabilized by three major components: Articular surfaces, ligaments, and musculotendinous units [27]. When we talk about dynamic protection of joints (stiffness generated by musculotendinous units [27]), not only concentric function of muscles should be considered, but also eccentric functions as well [27].

The mechanism that usually leads to an ankle sprain is an exaggerated inversion and supination of the foot [6]. Thus, it is logical to expect that the stronger the concentric function of the muscles that antagonize this exaggerated motion is, the less likely it will be to have an ankle sprain. Additionally, the stronger the eccentric contraction of supinators and invertors is, the fewer injuries will occur. Besides, it is reasonable to hypothesize that the faster these muscles activate, the less will be the incidence of this injury.

Even though Beynnon's group had conflicting results $[22,28]$, a more recent systematic review with meta-analysis is opening a door to clarify the role of muscle strength in ankle sprain [26]. This study is concordant with the theory exposed and showed that a decreased slow eccentric inversion strength would increase the incidence of ankle sprains [26].

Hagen, et al. [29] studied the correlation between the pronator-to-supinator-ratio (PSR) and ankle sprains in football and handball players. A higher PSR implies that pronator muscles are stronger than supinator muscles, which would, according to the mechanism above, reduce the incidence of ankle sprains. This study showed that a lower PSR is a risk factor for ankle sprain [29], supporting the theory above.

Peroneus longus and peroneus brevis are foot evertors that play a role in primary defense against ankle sprains $[30,31]$. According to the theory explained previously, as strong as these muscles are, the less likely would be to sustain an ankle sprain [32]. In fact, a recent study showed that peroneus longus weakness while walking on a ramp with more than $10^{\circ}$ slope increases the incidence of sprained ankles [32]. However, no significant relation was found for peroneus brevis [32].

Even though most would only consider the muscles that cross the ankle-complex when it comes to relate muscle strength and sprained ankles, a recent study [11] showed that an increase in hip strength, and even in core strength [33], might reduce the incidence of ankle sprains. This relation is based on kinetic chain theory, which postulate that impaired proximal function increases the likelihood of uncontrolled joint displacements throughout the lower kinetic chain [34]. Additionally, during landing (an ankle sprain risk condition where the ankle transits from unloaded to loaded [35]), the hip extensors muscles play an important role in the deceleration of the body [36], reinforcing the role of proximal muscles in ankle stabilization.

This information proposes a relation between ankle-complex muscles and ankle sprains. In fact, even proximal muscles might play a role in this injury. However, prospective studies that measure muscle strength of all the ankle-complex muscles should be developed, in order establish independent and clear risk factors.

When it comes to muscle-reaction time, the few existent literature doesn't support the theory proposed above. Kobayashi, et al. [26] found that earlier reaction of the peroneus brevis increases this injury. No relation was found for peroneus longus and tibialis anterior [26]. These results do not support the muscle-reaction time hypothesis. Nonetheless, as exposed by the authors, there is a possibility of beta-error [26]. Additionally, other study didn't find any significant relation between muscle-reaction time and ankle sprain [22]. More studies are required to verify this theory. 
Previous ankle sprain: At the beginning of this century, Beynnon's group [12] had already pointed previous ankle sprain as one of the most studied risk factors for a new injury. Even tough, at that point, and using the author's words, the literature was "divided". On one hand, one of the first prospective studies [37] studied 124 soccer athletes and found that those who suffered a prior ankle-ligament sprain were at a higher risk of sustaining the injury again; on the other hand, other studies revealed no increased risk for ankle sprain after sustaining a prior injury [12]. Beynnon's group suggested that the joint condition after injury depends on the associated damage to the ligaments and even the quality of the rehabilitation [12]. These reasons might be a possible justification for these divergent findings.

Recent studies reported previous ankle sprain as a risk factor for ipsilateral $[17,25]$ and contralateral [38] future lateral ankle injury. Moreover, a systematic review [39] identified and reviewed 26 articles, describing the relationship between injury and re-injury for the four most common lower extremity injury. Their results showed that previous ankle sprain is the most common injury resulting in a secondary sprain of the ipsilateral or contralateral ankle, which may be explained by neuromuscular factors [39]. Even though this research applies only to the active adult population, it gives a great support on how a previous sprained ankle might contribute to a future injury.

In our opinion, previous lateral ankle ligaments sprain as a risk factor for re-injury might be one of the risk factors with more scientific evidence supporting it.

Balance, neuromuscular control and proprioception: Proprioception is a complex process which is coordinated by afferent and efferent impulses, working as feedback loops [40]. It implies a fine tune between nervous and musculoskeletal systems, allowing one to be aware of his own joint position (joint position sense) and joint movement (kinesthesia) $[41,42]$; This neuromuscular feedback mechanism might be interrupted with injury and abnormalities, but surgical intervention and rehabilitation might restore it [42].

Proprioceptive rehabilitation training includes exercises that challenge the ability of the targeted joint to detect and react to afferent input regarding joint position, for example, balance exercises [43]. As this type of exercises and neuromuscular control are implied in proprioception, it's logical that different authors have explored the effectiveness of improving these diverse components in injury prevention, namely in the ankle joint.

As a matter of fact, when relating proprioceptive training and the incidence of ankle injuries, many authors suggested that an increase in proprioception mainly reduces the incidence of recurrent ankle sprain [43-47]. However, a recent review [47] showed that proprioceptive training programs were effective in reducing the incidence rates of ankle sprains in athletic population, including those with and those without a history of ankle sprains.

Concerning balance training, there are plenty of studies showing that balance-based exercises reduce the incidence of primary and recurrent ankle sprain [48-55]. Moreover, balance training increases joint position sense, dynamic neuromuscular control and postural sway [50]. According to different studies, an increase in these capabilities can reduce ankle sprains $[3,44,55-59]$.

There are even studies showing how multi-intervention programs are effective reducing the incidence of ankle sprain, namely by $50 \%$ [51]. However, the relative importance of each component of these programs remains unknown and warrants further research [46].

Different authors studied different proprioception related concepts and tried to relate them with ankle sprain incidence. Besides some studies have some cons, such as selection bias or the lack of prospective analysis, in our opinion, it's clear how the different components of proprioception affect the ankle joint. Further prospective research should be done, to determine the relative importance of each one of proprioception components and, ultimately, define proper prophylactic exercised-based programs.

Limb dominance: The only review of our knowledge that covered this topic, found no conclusive results [12]. There were studies stating that limb dominance was unrelated to ankle injury risk [22,60], while others showed that $92 \%$ of ankle injuries affected the dominant leg [37]. Further research with proper sample size is required, as these inconclusive findings might have been the result of different study designs and methods.

Foot type and other anatomic specificities (joint laxity, anatomic alignment and ankle-foot range of motion): Beynnon's, et al. review [12] was the only one we found relating anatomic foot type (pronated, supinated and neutral) and ankle sprains. However, no correlation was found between foot type and this injury. According to the authors, the reason behind these findings could be an inadequate anatomical classification system of the foot [12]. The typical classification is determined while the athlete is standing barefoot and not on an at-risk situation; additionally, this approach has not been related to musculoskeletal abnormalities, neither can identify foot biomechanics' defects [12]. A more recent and validated method [61], the foot posture index (FPI), also found no relation between anatomical foot type and ankle sprain, as there was no association between FPI score and the occurrence of this injury [62].

According to the previous referred limitations of foot type static measurement, dynamic methods that can be used on at-risk situations for sprained ankles should be developed. Moreover, future research should focus on determining these new methods specificity and sensitivity in predicting an ankle sprain, eventually leading to the identification of new risk factors for this injury.

Generalized joint and ankle-joint laxity, anatomic alignment and range of motion of the ankle-foot complex were covered on Beynnon's, et al. review [12]. However, none of these appeared to have a clear relation to ankle sprains. Future research is needed to clarify these topics.

\section{Extrinsic Risk Factors}


Ankle bracing and taping: Beynnon's, et al. [12] stated that braces were effective for reducing the risk of reinjuring an ankle, but not for a first-time sprain. Only considering the mechanical support given by bracing, one would expect that both athletes, with and without history of previous ankle sprain, had fewer injuries. Only athletes with previous ankle sprains had a reduction in the incidence of this injury, which suggests that bracing protects the joint, not only mechanically, but by fixing an issue in a previously injured ankle. Furthermore, the authors found no difference in the severity of ankle sprains with or without the use of the brace. Once again, considering only brace mechanical support, it was expected that the braced group had less injuries, but with higher severity. This would happen, because an injury would only occur when the generated tension surpassed the brace mechanical threshold. A more recent systematic review [63] found a $69 \%$ reduction in ankle sprains among athletes who wore braces and had an history of previous ankle sprain. Even though bracing effectively decreased the incidence of ankle sprains, the exact mechanism behind it was still controversial.

A recent systematic review [48] concluded that athletes who wore braces had fewer ankle sprains $(p<0.05)$ and reduced the risk of sprains by $64 \%$, either if they had or hadn't a previous sprain history. It's more likely that this systematic review had less bias than the ones mentioned above, considering the larger number of studies included. According to Karlsson, et al. [64], reduction of mechanical ankle instability, limitation of extremes of ankle motion, as well as an increase in proprioception, would contribute to the increased protection given by external ankle supports. Moreover, the best protection is found in ankles with the highest degree in mechanical instability [64], which might explain the results found in the reviews above $[12,48]$. A recent systematic review [52] also states that braces effects are due to mechanical and neuromuscular enhancements. Nonetheless, there is a review [41] that found no effect of external ankle supports on proprioception, and even suggested that proprioception may be worst in some movement planes. However, they didn't discourage the use of braces and tapes [41].

Another question that is often raised, is if there is any difference between bracing or taping the ankle when it comes to reduce the incidence of ankle sprains. Current evidence is mixed whether bracing or taping is more effective in reducing ankle injury risk [52]. Even though we found a study suggesting that braces are superior to tape [65], most of the authors suggested no difference between either one of the external ankle supports $[58,63,66]$. However, any potential prophylactic effect should be balanced against the baseline risk of the activity, the supply and cost of the particular device, and the perceived loss of performance [66] (loss of performance will only occur if the brace/tape isn't appropriately applied [67]).

In our opinion, braces and tapes are equally effective reducing the incidence of ankle sprains in athletes with or without history of previous injury. However, the exact mechanism behind these external ankle supports it's not yet clearly understood. More researches are needed to clarify the current mixed evidence about this topic.
Shoe type: A systematic review from the late 90's relating athlete's shoe type and ankle sprain prevention reported numerous findings [67]. High top shoes were recommended for injury prevention in basketball and football [67]. However, lower incidences of sprained ankles were found in athletes wearing specially designed swivel shoes instead of the conventional cleats [67]. A recent systematic review with meta-analysis found that high top shoes reduce the risk of ankle injury in basketball players [52]. Further studies should be developed to clarify if the characteristics of the shoe in the correspondent sport correlate with ankle sprains.

Sport category and floor type: We found two systematic reviews relating sports category and the floor type with the incidence of ankle sprains [1,5]. Both stated that the incidence of sprained ankles was higher in indoor, court and team sports. The nature these sports might explain the higher incidence of injury on this floor type, likely because these are sports with high-demand to the ankle [2-4].

Player position: Different authors found no difference in risk of ankle injury among player positions [12]. However, we found a recent study [68] that video-analyzed ankle injuries in world-class volleyball and stated that blockers had the highest incidence of injury, mainly due to landing on an attacker that crossed to the opponent's court. This fact might raise the need to review volleyball game rules.

Hence, according to different sports specificities, the player position might be a risk factor for ankle sprains. A thorough analysis of different in-game situations is required to establish such relation.

\section{Conclusion}

Most professionals working with athletes would rather prevent an injury than treat one. However, solid scientific evidence is needed to build structured and efficient prevention programs. Unfortunately, despite the high incidence of ankle sprain, the literature hasn't reached a consensus when it comes to define clear risk factors for this injury. Similarly to others, our review found previous ankle sprain as a risk factor for a new injury, and external ankle support (bracing/taping) as a preventive measure for a new or recurrent sprained ankle. Moreover, our review suggests that ankle sprains might be related to specific ankle-complex muscle strength, and even with proximal muscle strength. It also appears that balance and proprioceptive training might reduce the incidence of this injury. Athletes practicing indoor and court team sports also have a higher risk of sustaining a sprained ankle. Even though the next topics were less unanimous, it appears that younger female athletes, with a high BMI, might have an increased risk for ankle sprain. Shoe type and player position might also be a risk factor in specific sports. We didn't find any correlation between muscle reaction time nor anatomic foot type and ankle sprains.

To our knowledge, we were the only ones in more than fifteen years approaching these many ankle sprain risk factors in the same review. We paralleled different studies, from different authors and years, and tried to understand how a specific risk factor relates with this injury. 
Even though there are a few clearly defined risk factors for ankle sprain, further prospective studies are needed to properly evaluate each suggested risk factor for this injury, reducing the actual bias and uncertainty. It's mandatory to clear if some of the results found are reproducible, or if they're due to confounders. However, it seems that the correct way has started to be paved. After defining clear risk factors for ankle sprains, it will be possible to create proper education and prevention strategies.

\section{References}

1. Fong DT, Hong Y, Chan LK, et al. (2007) A systematic review on ankle injury and ankle sprain in sports. Sports Med 37: 73-94.

2. Quigley TB (1959) Management of ankle injuries sustained in sports. J Am Med Assoc 169: 1431-1436.

3. Grassi A, Alexiou K, Amendola A, et al. (2018) Postural stability deficit could predict ankle sprains: A systematic review. Knee Surg Sports Traumatol Arthrosc 26: 3140-3155.

4. G McKay, P Goldie, W Payne, et al. (2001) Ankle injuries in basketball: Injury rate and risk factors. Br J Sports Med 35: 103-108.

5. Doherty C, Delahunt E, Caulfield B, et al. (2014) The incidence and prevalence of ankle sprain injury: A systematic review and meta-analysis of prospective epidemiological studies. Sports Med 44: 123-140.

6. Cox JS (1985) Surgical and nonsurgical treatment of acute ankle sprains. Clin Orthop Relat Res 198: 118-126.

7. Tiemstra JD (2012) Update on acute ankle sprains. Am Fam Physician 85: 1170-1176.

8. Diamond JE (1989) Rehabilitation of ankle sprains. Clin Sports Med 8: 877-891.

9. Kerkhoffs GM, van den Bekerom M, Elders LA, et al. Diagnosis, treatment and prevention of ankle sprains: An evidence-based clinical guideline. Br J Sports Med 46: 854-860.

10. Henry JH, B Lareau, D Neigut (1982) The injury rate in professional basketball. Am J Sports Med 10: 16-18.

11. De Ridder R, Witvrouw E, Dolphens M, et al. (2017) Hip strength as an intrinsic risk factor for lateral ankle sprains in youth soccer players: A 3-season prospective study. Am J Sports Med 45: 410416.

12. Beynnon BD, DF Murphy, DM Alosa (2002) Predictive factors for lateral ankle sprains: A literature review. J Athl Train 37: 376380.

13. JGP Williams (1971) Aetiological classification of injuries in sportsmen. Br J Sports Med 4: 228-230.

14. Hølmer P, Søndergaard L, Konradsen L, et al. (1994) Epidemiology of sprains in the lateral ankle and foot. Foot Ankle Int 15: 72-74.

15. Pefanis N, Papaharalampous X, Tsiganos G, et al. (2009) The effect of $Q$ angle on ankle sprain occurrence. Foot Ankle Spec 2: $22-26$.

16. Waterman BR, Owens BD, Davey S, et al. (2010) The epidemiology of ankle sprains in the United States. J Bone Joint Surg Am 92: 2279-2284.

17. Kofotolis ND, E Kellis, SP Vlachopoulos (2007) Ankle sprain injuries and risk factors in amateur soccer players during a 2-year period. Am J Sports Med 35: 458-466.

18. Quatman-Yates CC, Quatman CE, Meszaros AJ, et al. (2012) A systematic review of sensorimotor function during adolescence: A developmental stage of increased motor awkwardness? $\mathrm{Br} \mathrm{J}$ Sports Med 46: 649-655.

19. Lohmander LS, Ostenberg A, Englund M, et al. (2004) High prevalence of knee osteoarthritis, pain, and functional limitations in female soccer players twelve years after anterior cruciate ligament injury. Arthritis Rheum 50: 3145-3152.

20. Valderrabano V, Hintermann B, Horisberger M, et al. (2006) Ligamentous posttraumatic ankle osteoarthritis. Am J Sports Med 34: 612-620.

21. Milgrom C, Shlamkovitch N, Finestone A, et al. (1991) Risk factors for lateral ankle sprain: A prospective study among military recruits. Foot Ankle 12: 26-30.

22. Beynnon BD, Renström PA, Alosa DM, et al. (2001) Ankle ligament injury risk factors: A prospective study of college athletes. J Orthop Res 19: 213-220.

23. Willems TM, Witvrouw E, Delbaere K, et al. (2005) Intrinsic risk factors for inversion ankle sprains in male subjects: A prospective study. Am J Sports Med 33: 415-423.

24. McHugh MP, Tyler TF, Tetro DT, et al. (2006) Risk factors for noncontact ankle sprains in high school athletes: The role of hip strength and balance ability. Am J Sports Med 34: 464-470.

25. Tyler TF, McHugh MP, Mirabella MR, et al. (2006) Risk factors for noncontact ankle sprains in high school football players: The role of previous ankle sprains and body mass index. Am J Sports Med 34: 471-475.

26. Kobayashi T, Tanaka M, Shida M (2016) Intrinsic risk factors of lateral ankle sprain: A systematic review and meta-analysis. Sports Health 8: 190-193.

27. Hertel J (2002) Functional anatomy, pathomechanics, and pathophysiology of lateral ankle instability. J Athl Train 37: 364-375.

28. Baumhauer JF, Alosa DM, Renström AF (1995) A prospective study of ankle injury risk factors. Am J Sports Med 23: 564-570.

29. Hagen M, Asholt J, Lemke M, et al. (2016) The angle-torquerelationship of the subtalar pronators and supinators in male athletes: A comparative study of soccer and handball players. Technol Health Care 24: 391-399.

30. Konradsen L, M Voigt, C Hojsgaard (1997) Ankle inversion injuries. The role of the dynamic defense mechanism. Am J Sports Med 25: 54-58.

31. Louwerens JW, van Linge B, de Klerk LW, et al. (1995) Peroneus longus and tibialis anterior muscle activity in the stance phase. A quantified electromyographic study of 10 controls and 25 patients with chronic ankle instability. Acta Orthop Scand 66: 517-523.

32. Lee SY, Lee SM, Jung JM (2017) Peroneus longus activity according to various angles of a ramp during cross-ramp walking and onelegged standing. J Back Musculoskelet Rehabil 30: 1215-1219.

33. Stearns KM, CM Powers (2014) Improvements in hip muscle performance result in increased use of the hip extensors and abductors during a landing task. Am J Sports Med 42: 602-609.

34. Chuter VH, XA Janse de Jonge (2012) Proximal and distal contributions to lower extremity injury: A review of the literature. Gait Posture 36: 7-15.

35. Stormont DM, Morrey BF, An KN, et al. (1985) Stability of the loaded ankle. Relation between articular restraint and primary and secondary static restraints. Am J Sports Med 13: 295-300. 
36. Powers CM (2010) The influence of abnormal hip mechanics on knee injury: A biomechanical perspective. J Orthop Sports Phys Ther 40: 42-51.

37. Ekstrand J, J Gillquist (1983) Soccer injuries and their mechanisms: A prospective study. Med Sci Sports Exerc 15: 267-270.

38. Hiller CE, Refshauge KM, Herbert RD, et al. (2008) Intrinsic predictors of lateral ankle sprain in adolescent dancers: A prospective cohort study. Clin J Sport Med 18: 44-48.

39. Fulton J, Wright K, Kelly M, et al. (2014) Injury risk is altered by previous injury: A systematic review of the literature and presentation of causative neuromuscular factors. Int J Sports Phys Ther 9: 583-595.

40. Postle K, D Pak, TO Smith (2012) Effectiveness of proprioceptive exercises for ankle ligament injury in adults: A systematic literature and meta-analysis. Man Ther 17: 285-291.

41. Raymond J, Nicholson LL, Hiller CE, et al. (2012) The effect of ankle taping or bracing on proprioception in functional ankle instability: A systematic review and meta-analysis. J Sci Med Sport 15: 386-392.

42. Lephart SM, Pincivero DM, Giraldo JL, et al. (1997) The role of proprioception in the management and rehabilitation of athletic injuries. Am J Sports Med 25: 130-137.

43. Schiftan GS, LA Ross, AJ Hahne (2015) The effectiveness of proprioceptive training in preventing ankle sprains in sporting populations: A systematic review and meta-analysis. J Sci Med Sport 18: 238-244.

44. de Noronha M1, Refshauge KM, Herbert RD, et al. (2006) Do voluntary strength, proprioception, range of motion, or postural sway predict occurrence of lateral ankle sprain? Br J Sports Med 40: 824-828.

45. Porter T, A Rushton (2015) The efficacy of exercise in preventing injury in adult male football: A systematic review of randomised controlled trials. Sports Med Open 1: 4.

46. Osborne MD, TD Rizzo Jr (2003) Prevention and treatment of ankle sprain in athletes. Sports Med 33: 1145-1150.

47. Rivera MJ, Winkelmann ZK, Powden CJ, et al. (2017) Proprioceptive training for the prevention of ankle sprains: An evidence-based review. J Athl Train 52: 1065-1067.

48. Bellows R, CK Wong (2018) The effect of bracing and balance training on ankle sprain incidence among athletes: A systematic review with meta-analysis. Int J Sports Phys Ther 13: 379-388.

49. van der Wees PJ, Lenssen AF, Hendriks EJ, et al. (2006) Effectiveness of exercise therapy and manual mobilisation in ankle sprain and functional instability: A systematic review. Aust J Physiother 52: 27-37.

50. De Vasconcelos GS, Cini A, Sbruzzi G, et al. (2018) Effects of proprioceptive training on the incidence of ankle sprain in athletes: Systematic review and meta-analysis. Clin Rehabil 32: 1581-1590.

51. Hubscher M, Zech A, Pfeifer K, et al. (2010) Neuromuscular training for sports injury prevention: A systematic review. Med Sci Sports Exerc 42: 413-421.

52. Taylor JB, Kevin R Ford, Anh-Dung Nguyen, et al. (2015)
Prevention of lower extremity injuries in basketball: A systematic review and meta-analysis. Sports Health 7: 392-398.

53. McKeon PO, J Hertel (2008) Systematic review of postural control and lateral ankle instability, part I: Can deficits be detected with instrumented testing. J Athl Train 43: 293-304.

54. McKeon PO, J Hertel (2008) Systematic review of postural control and lateral ankle instability, part II: Is balance training clinically effective? J Athl Train 43: 305-315.

55. de la Motte SJ, Lisman P, Gribbin TC, et al. (2019) Systematic review of the association between physical fitness and musculoskeletal injury risk: Part 3 - flexibility, power, speed, balance, and agility. J Strength Cond Res 33: 1723-1735.

56. Emery CA, WH Meeuwisse (2010) The effectiveness of a neuromuscular prevention strategy to reduce injuries in youth soccer: A cluster-randomised controlled trial. $\mathrm{Br} J$ Sports Med 44: 555-562.

57. de Vries JS, Krips R, Sierevelt IN, et al. (2011) Interventions for treating chronic ankle instability. Cochrane Database Syst Rev.

58. Verhagen EA, K Bay (2010) Optimising ankle sprain prevention: A critical review and practical appraisal of the literature. $\mathrm{Br} J$ Sports Med 44: 1082-1088.

59. Dallinga JM, A Benjaminse, KA Lemmink (2012) Which screening tools can predict injury to the lower extremities in team sports? A systematic review. Sports Med 42: 791-815.

60. Surve I, Schwellnus MP, Noakes T, et al. (1994) A fivefold reduction in the incidence of recurrent ankle sprains in soccer players using the sport-Stirrup orthosis. Am J Sports Med 22: 601-606.

61. Keenan AM, Redmond AC, Horton M, et al. (2007) The foot posture index: Rasch analysis of a novel, foot-specific outcome measure. Arch Phys Med Rehabil 88: 88-93.

62. Cherati AS, M Dousti, S Younespour (2016) Association between foot posture index and ankle sprain in indoor football players. Glob J Health Sci 8: 51426.

63. Dizon, JM, JJ Reyes (2010) A systematic review on the effectiveness of external ankle supports in the prevention of inversion ankle sprains among elite and recreational players. J Sci Med Sport 13: 309-317.

64. Karlsson J, L Sward, GO Andreasson (1993) The effect of taping on ankle stability. Practical implications. Sports Med 16: 210-215.

65. Vuurberg G, Hoorntje A, Wink LM, et al. (2018) Diagnosis, treatment and prevention of ankle sprains: Update of an evidence-based clinical guideline. Br J Sports Med 52: 956.

66. Handoll HH, Rowe BH, Quinn KM, et al. (2001) Interventions for preventing ankle ligament injuries. Cochrane Database Syst Rev.

67. Thacker SB, Stroup DF, Branche CM, et al. (1999) The prevention of ankle sprains in sports. A systematic review of the literature. Am J Sports Med 27: 753-760.

68. Skazalski C, Kruczynski J, Bahr MA, et al. (2018) Landing-related ankle injuries do not occur in plantarflexion as once thought: A systematic video analysis of ankle injuries in world-class volleyball. Br J Sports Med 52: 74-82.

DOI: $10.36959 / 987 / 247$

Copyright: (C) 2019 Correia MAC, et al. This is an open-access article distributed under the terms of the Creative Commons Attribution License, which permits unrestricted use, distribution, and reproduction in any medium, provided the original author and source are credited. 